\title{
SOME RINGS OF DIFFERENTIAL OPERATORS WHICH ARE MORITA EQUIVALENT TO THE WEYL ALGEBRA $A_{1}$
}

\author{
IAN M. MUSSON
}

ABSTRACT. For a certain class of semigroup algebras $k \Lambda$, we show that the ring of all differential operators on $k \Lambda$ is Morita equivalent to the first Weyl algebra $A_{1}$.

Let $k$ be a field, and $K$ a commutative $k$-algebra. We denote by $D_{0}(K, K)$ the set of $K$-linear maps $K \rightarrow K$, and if $p \geqslant 1$ then a $k$-linear map $f: K \rightarrow K$ belongs to $D_{p}(K, K)$ provided the map $[f, r]$ defined by $[f, r](s)=f(r s)-r f(s)$ for $s \in K$ belongs to $D_{p-1}(K, K)$ for all $r \in K$. The set $D(K)=\bigcup_{p \geqslant 0} D_{p}(K, K)$ forms a subring of $\operatorname{End}_{k}(K)$ called the ring of differential operators on $K$. We consider $K$ as a left $D(K)$-module where $f \cdot r=f(r)$ for all $f \in D(K), r \in K$.

In this note we show that for certain subalgebras $K$ of $k[t]$, where $k$ is a field of characteristic zero and $t$ an indeterminate, the ring $D(K)$ is Morita equivalent to the Weyl algebra $A_{1}$. If $\Lambda$ is a subsemigroup of the set of nonnegative integers $\mathbf{N}$, we let $k \Lambda$ be the semigroup algebra on $\Lambda$, which we regard as the subalgebra of $k[t]$ spanned by $\left\{t^{\lambda} \mid \lambda \in \Lambda\right\}$.

THEOREM. If the semigroup algebra $k \Lambda$ has the same quotient field as $k[t]$, then $D(K)$ is Morita equivalent to $A_{1}$.

The present proof is due to S. P. Smith.

The first result is well known, see [2, Lemma 2].

LEMMA 1. If $K$ is a $k$-algebra and $C$ a multiplicatively closed subset of $K$ consisting of regular elements, then any differential operator on $K$ has a unique extension to $K_{C}$. Hence $D(K)$ may be regarded as a subalgebra of $D\left(K_{C}\right)$. In addition,

$$
D(K)=\left\{x \in D\left(K_{C}\right) \mid x \cdot K \subseteq K\right\} .
$$

We let $A_{1} \doteq k[q, p]$ be the $k$-algebra generated by $q$ and $p$ subject to the relation $p q-q p=1$, and regard $A_{1}$ as $D(k[t])$ where $q$ acts as a multiplication by $t$ and $p$ as $d / d t$. If $K$ is a subalgebra of $k[t]$ with the same quotient field $k(t)$, then by Lemma 1 , we can view both $D(K)$ and $A_{1}$ as subalgebras of the quotient ring $Q$ of $A_{1}$. We set $I=\left\{x \in A_{1} \mid x \cdot k[t] \subseteq K\right\}$ and $S=\{x \in Q \mid x I \subseteq I\}$. Then $S \cong$ End $_{A_{1}}(I)$, see [1, p. 69].

LEMMA 2. $D(K) \subseteq S$.

Received by the editors September 4, 1985.

1980 Mathematics Subject Classification (1985 Revision). Primary 16A45

Key words and phrases. Rings of differential operators, Morita equivalence, Weyl algebra. 
Proof. If $x \in D(K)$, then $x$ acts on $k(t)$. Also $(x I) k[t]=x(I k[t]) \subseteq x K \subseteq K$. Therefore, $x I \subseteq I$ and so $x \in S$.

Proof OF THE TheOrem. We now assume $K=k \Lambda$ is a semigroup algebra contained in $k[t]$ with quotient field $k(t)$. Since $k[t] / K$ is a finitely generated $K$-module which is torsion with respect to $C=\left\{t^{\lambda} \mid \lambda \in \Lambda\right\}$, it follows that $t^{n} k[t] \subseteq$ $K$ for some $n \geqslant 0$. Hence the set $\Lambda^{\prime}=\mathbf{N} \backslash \Lambda$ is finite. We set $K^{\prime}=\bigoplus_{\lambda \in \Lambda^{\prime}} k t^{\lambda}$ so $K^{\prime}$ is a subspace of $k[t]$ with $k[t]=K \oplus K^{\prime}$. For all $\lambda \in \mathbf{N}$, we have $q p \cdot t^{\lambda}=\lambda t^{\lambda}$. If we set $f(q p)=\Pi_{\lambda \in \Lambda^{\prime}}(q p-\lambda)$, then $f(q p) \cdot K^{\prime}=0$, so $f(q p) \in I$. Also for $\lambda \in \Lambda, f(q p) \cdot t^{\lambda}$ is a nonzero scalar multiple of $t^{\lambda}$, so $f(q p) \cdot K=K$. It follows that $I k[t]=K$.

We claim that $S \subseteq D(K)$. If $t^{n} k[t] \subseteq K$ as above, then $q^{n} \in I$, so if $x \in S$, then $x q^{n} \in I \subseteq A_{1}$. Since $C^{\prime}=\left\{q^{m} \mid m \geqslant 0\right\}$ is an Ore set in $A_{1}, \quad x \in\left(A_{1}\right)_{C^{\prime}}=$ $k\left[q, q^{-1}, p\right]=D\left(k\left[t, t^{-1}\right]\right)$. Therefore, $S$ acts on $k\left[t, t^{-1}\right]$. Also, if $x \in S$, then $x \cdot K=x \cdot(I k[t])=(x I) \cdot k[t] \subseteq I \cdot k[t] \subseteq K$. Hence $x \in D(K)$. We have shown that $D(K)=S$, and $S$ is isomorphic to the endomorphism ring of the right ideal $I$ of $A_{1}$. Since $A_{1}$ is hereditary by [3], and simple, $I$ is a progenerator. Therefore, $D(K)$ is Morita equivalent to $A_{1}$.

\section{REFERENCES}

1. David Eisenbud and J. C. Robson, Modules Over Dedekind prime rings, J. Algebra 16 (1970), 67-85.

2. R. Hart, Differential operators on affine algebras, J. London Math. Soc. (2), 28 (1983), 470-476.

3. George S. Rinehart, Note on the global dimension of a certain ring, Proc. Amer. Math. Soc. 13 (1962), 341-346.

Department of Mathematical Sciences, University of Wisconsin - Milwaukee, Milwaukee, WISCONSIN 53201 